\title{
Aplicación del método sistémico al diseño de un modelo conceptual para sistemas integrales de gestión QHSE3+ en PYMES
}

\author{
Poveda-Orjuela, Pedro Pablo ${ }^{a}$; García-Díaz, Juan Carlos ${ }^{b}$ \& Hernandis-Ortuño, Bernabéc
}

${ }^{a}$ Industrial Engineering Department, Universidad del Norte, Barranquilla, Colombia.ppoveda@uninorte.edu.co.

${ }^{b}$ Centre for Quality and Change Management, Universitat Politècnica de València, España. juagardi@eio.upv.es.

${ }^{c} \mathrm{PhD}$, Full time Professor, Universitat Politècnica de Valencia, España. bhernand@degi.upv.es.

\section{Resumen}

La alta vulnerabilidad y el elevado porcentaje de fracasos y cierres de las PYME, pone en evidencia la necesidad de diseñar un modelo que con un enfoque básico funcional e integral de riesgos y calidad, permita a los actores de las PYME conocer, apropiar y aplicar las Buenas Prácticas en los ciclos de Planificación, Operación, Control y Mejora de sus productos, procesos y negocios. Los autores presentan en este documento el enfoque y la aplicación del Método Sistémico en el Diseño de un Modelo Conceptual para los Sistemas de Gestión Integral de las PYMEs, configurado a partir de la conjugación de las Buenas Prácticas relacionadas con:

i) El Ciclo de Gestión de Riesgos Estratégicos y Operacionales,

ii) La Efectividad en el Manejo de los Procesos Directivos, de la Cadena de Valor y de Apoyo.

iii) El éxito sostenible del negocio PYME, entendido desde la eco eficiencia, la competitividad y la rentabilidad.

Las Buenas Prácticas se direccionan desde el cuerpo del conocimiento contenido en la familia de normas (ISO 31000:2009)"Risk Management. Principles and Guidelines", al igual que en los referenciales para la Gestión de Calidad Q(ISO 9001:2015), Salud y Seguridad HS(ISO DIS 45000:2016), Gestión Ambiental E(ISO 14001:2015), Gestión para la Eficiencia Energética E2(ISO 50001:2011); y de otras componentes de riesgo, ligadas a la conformación del acrónimo QHSE3+, asociado a la integración de las iniciales en ingles señaladas en negrita previamente, según se requiera.

El modelo ha sido objeto de realimentación y de una validación preliminar mediante su aplicación en varias PYME del Caribe Colombiano, desde actividades de consultoría, y con grupos de investigación en asignaturas de Posgrado.

Los resultados obtenidos permiten:

i) Ratificar la utilidad e importancia de poner a disposición de los emprendedores un Modelo con Instrumentos Básicos para la Planificación y la Gestión Integral de Riesgos de afectación de la Calidad, la Salud y la Seguridad, el Medio Ambiente, 
los Recursos y el Desempeño Energéticos, al igual que otras componentes particulares de riesgos aplicables al tema particular de cada negocio PYME.

ii) Destacar el beneficio asociado a disponer de instrumentos sencillos de Gestión Integral de Riesgos QHSE3+ que contribuyan en el éxito sostenible y la competitividad de los negocios PYME.

iii) Demostrar con valoraciones especificas, que mediante la aplicación del Modelo y sus instrumentos de Planificación y Operación, es posible traducir el éxito sostenible, en la disminución de la vulnerabilidad estratégica global y de cada proceso, con magnitudes entre el 15\% y el 37\%, que pueden llegar a representar cifras mayores de reducción de costos, en la medida en que se apropien e institucionalicen los métodos y sus principios asociados.

Palabras clave: diseño, método sistémico, sistema integral de gestión, modelo conceptual, éxito sostenible, buenas prácticas.

\begin{abstract}
The high vulnerability and the high percentage of failures and closures of SMEs, reveal the need to develop a working model which should begin with a basic Integral and functional approach of risk and quality, and with easy application tools, that allow the SME's characters know, adopt and apply good practices in Planning cycles, operation, control and improvement of products, processes and businesses.

The authors present in this paper the focus and application of Systemic Method in Designing a Conceptual Model for Integrated Systems Management SMEs, configured from the combination of Good Practices related to:

i) The Management Cycle Strategic and Operational Risks,

ii) Effectiveness of the Governing Process Management, Value Chain and Support Services.

iii) Sustainable business success SMEs understood from the eco-efficiency, competitiveness and profitability.

These good practices are addressed from the body of knowledge contained in the standards family (ISO 31000:2009)"Risk Management. Principles and Guidelines", as in the standard for Quality Management Q(ISO 9001: 2015), Health and Safety HS(ISO DIS 45000: 2016), Environmental Management E(ISO 14001: 2015), Energy Efficiency E2(ISO 50001: 2011 ); and other risk components, linked to the formation of Acronym QHSE3+, associated with the integration of english initials previously indicated in bold, as required.The model and instruments associated have been object of feedback and validation through its application in several SME's, since the consulting activities, and with research trams in postgraduate courses from Universidad del Norte, in Barranquilla.
\end{abstract}

The results allow: 
i) To confirm the usefulness and importance of making available to entrepreneurs with a model with Basic Instruments for Planning and Integrated Risk Management affectation of Quality, Health and Safety, Environment, Resources and Energy Performance, like other individual risk components applicable to the particular theme of each business SMEs.

ii) Highlight the benefits associated with simple tools that have implemented the Integrated Risk Management QHSE3+ contribute to the strategic perspective of sustainable success and competitiveness of the SME business.

iii) Demonstrate with specific assessments, that by applying the Model and its instruments of planning and operation, it is possible to translate sustainable success in reducing global strategic vulnerability and each process, with magnitudes between $15 \%$ and $37 \%$, which may account for higher numbers of cost reduction, to the extent that ownership and institutionalize methods and its associated principles.

Keywords: design, systemic method, integrated management system, conceptual model, sustainable success, good practices.

\section{Introducción}

En todo el mundo es evidente la necesidad estratégica de fortalecer el núcleo de desarrollo y la gestión efectiva de los negocios que conforman el músculo empresarial y social más importante del planeta: las PYMEs, que además soportan una componente clave en la estrategia de crecimiento, racionalización de costos y desarrollo de muchos sectores de la economía: La Tercerización y la Especialización.

En contravía, se destaca la dura realidad de las estadísticas frías y contundentes, que nos señalan que mas del $80 \%$ de las PYME de los cinco continentes, se siguen cerrando por bancarrota antes de superar sus 5 años de existencia.

Las causas y consecuencias asociadas a esta situación, que pueden observarse en la Figura 1, estructurada mediante la aplicación del enfoque de la Metodología del Marco Lógico para Proyectos, (NACIONES UNIDAS - CEPAL, 2005), ponen de manifiesto la necesidad de desarrollar un modelo básico de trabajo para la gestión, que a partir de un enfoque integral básico, y con herramientas de fácil aplicación, permita a los actores de las PYME conocer, apropiar y aplicar Buenas Prácticas en los ciclos que tienen lugar durante la configuración, planificación desarrollo y consolidación de sus Proyectos de Emprendimiento y creación de PYMES, para garantizar el éxito sostenible de sus empresas. 
Aplicación del método sistémico al diseño de un modelo conceptual para sistemas integrales de gestión QHSE 3+ en PYMES

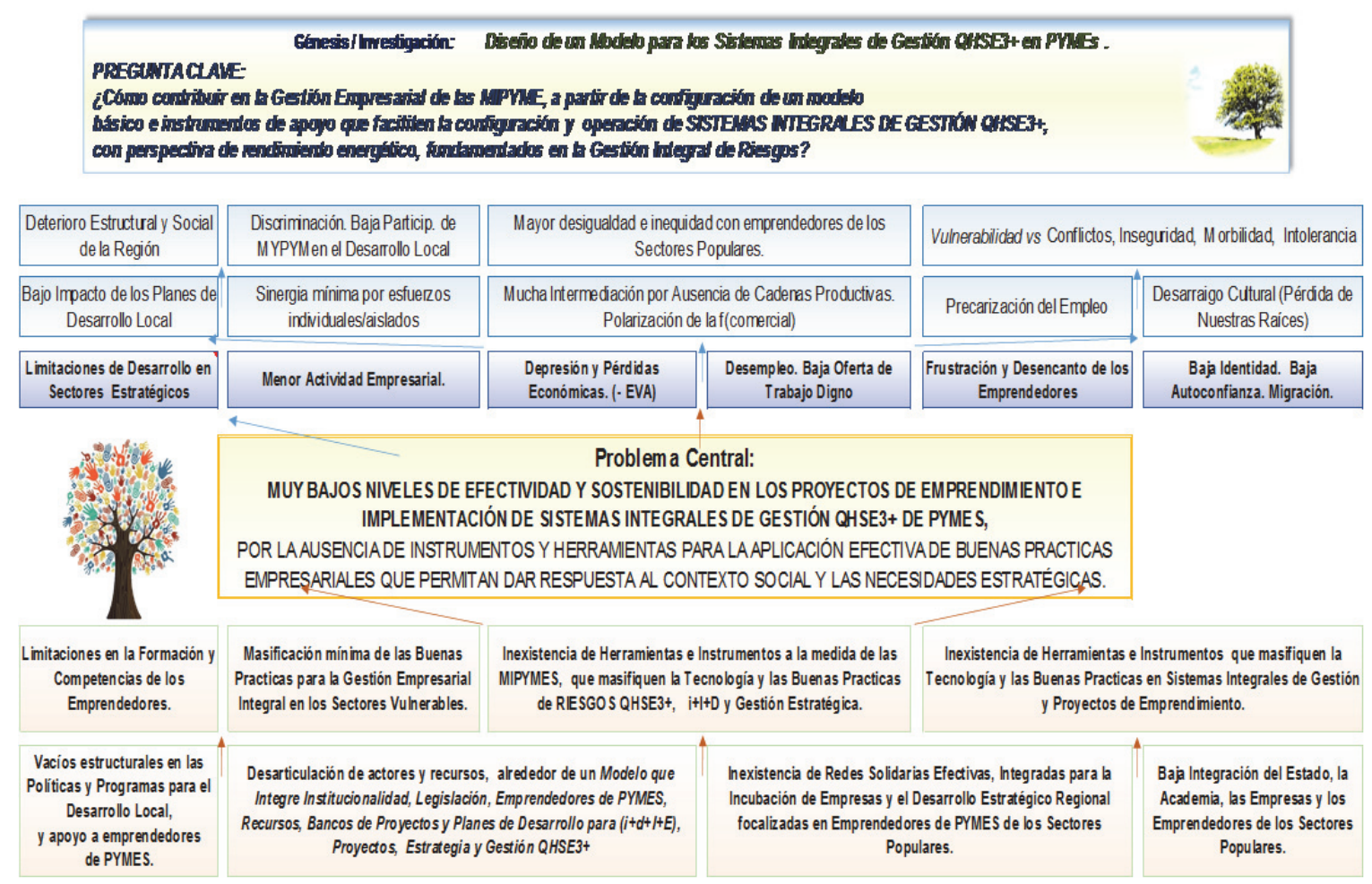

Fig. 1 Aplicación de la Metodología del Marco Lógico al Problema Objeto de la Investigación. Fuente: Realización de los Autores.

\section{Problemática y Objetivo.}

No obstante el desarrollo de los Programas de los Gobiernos de las diferentes latitudes, y los incentivos financieros que amplian los criterios de clasificación de las PYME para posibilitar un mayor acceso a los beneficios, como por ejemplo la aplicación flexible de la Recomendación C 20031422 (CEE, 2003), la dura realidad es que son demasiadas las barreras que desafían al espíritu emprendedor y a sus proyectos de construir y hacer empresa:

a) Las dificultades normales asociadas a tener un buen equipo de socios o un buen socio estable en el ámbito emocional, técnico, administrativo y financiero,

b) Las restricciones y limitaciones propias del mundo de hoy, en cuanto a los recursos más escasos y más costosos, la agudización de problemas ambientales como el acceso continuo al agua, la energía, los recursos naturales, o el calentamiento global, entre otros,

c) Las dificultades propias del mercado en su naturaleza puntual de oferta-demanda-rentabilidad, exclusivamente focalizada en el retorno del activo, y no en la generación integral de valor,

d) El vértigo del cambio externo en los intereses, gustos y necesidades de los clientes y el mercado, los proveedores, la legislación, la tecnología y a veces también las condiciones geopolíticas y macroeconómicas del entorno,

e) El imperativo del cambio interno de procesos, cultura y talentos para poder estar a tono y responder cuándo y cómo debe ser, a las condiciones y demanda del mercado, 
f) El costo del dinero, y las dificultades para lograr el fondeo de proyectos de emprendimiento, bajo tasas que no sean de usura, o que como mínimo esten por debajo de los niveles de rentabilidad después de impuestos, depreciaciones y otros deducibles.

g) Las limitaciones desde el punto de vista de competencias, experiencia, organización y métodos de administración de los Negocios y Proyectos PYME.

Con esta complejidad, el camino de la solución está determinado por el desarrollo de las competencias de los actores de los Proyectos PYME, y la aplicación de herramientas y enfoques que permitan tener una visión y un manejo integral y holístico del Negocio y de los Proyectos de Emprendimiento, fundamentado en la visualización y aplicación de las Buenas Prácticas, entendidas como las medidas de prevención y control que deben adoptar los emprendedores para reducir la vulnerabilidad ante el enjambre de riesgos, y soportar la toma de decisiones fundamentada en la inteligencia que permite conocer, y comprender qué está pasando, y prever qué puede ocurrir en los Proyectos de emprendimiento y en la operación e interacción del Negocio PYME con su entorno y contexto.

El objetivo del trabajo que describe este documento, tiene que ver precisamente con el aporte al esfuerzo emprendedor y el éxito sostenible de las PYMEs, a partir de la Aplicación del Método Sistémico al Diseño y Configuración de un Modelo Básico para la Planificación, Operación, Realimentación y Mejora de los Sistemas Integrales de Gestión, fundamentado en la Gestión Integral de Riesgos (Poveda Orjuela, et al., 2015-A), y en la aplicación de las Buenas Prácticas contenidas en las Normas Internacionales relacionadas con las componentes QHSE3+.

\section{Metodología para el Diseño y Configuración del Modelo propuesto.}

En el desarrollo de la investigación se conjuga la metodología de Investigación Aplicada, con la Investigación Cualitativa y Cuantitativa, requeridas para abordar, definir el problema, investigar, comprobar y/o rechazar/reformular las hipótesis que fundamentan el modelo conceptual, a partir del acercamiento retrospectivo al problema y el estudio de casos individuales, mediante diagnósticos, sondeos y observación directa. 
Aplicación del método sistémico al diseño de un modelo conceptual para sistemas integrales de gestión QHSE $3+$ en PYMES

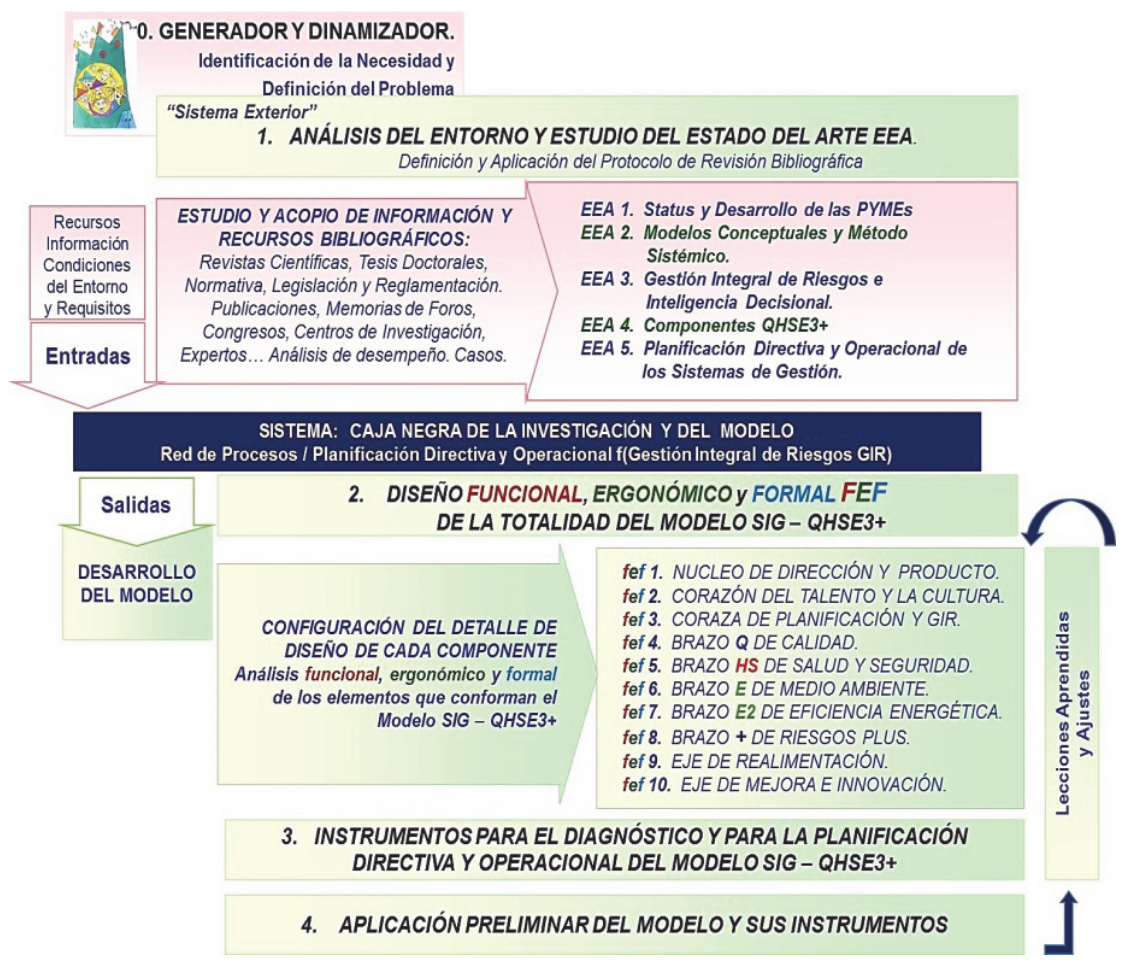

Fig.2 Metodología de la Investigación Aplicada para el desarrollo del Modelo SIG-QHSE3+. Fuente: Realización de los Autores.

Como se puede apreciar en la figura 2, una vez determinada la necesidad e identificado el problema, la metodología empleada considera las cuatro fases básicas, ligadas al flujo de la Investigación, que se enuncian a continuación:

\subsection{Definición y Aplicación del Protocolo de Revisión del Estado del Arte:}

El proceso de Revisión Bibliográfica para el Estudio del Estado del Arte, ha considerado la investigación, a partir de la definición de los cinco bloques que se relacionan a continuación, mediante la definición de temas, subtemas y preguntas y palabras clave que nos permiten determinar qué nos interesa en términos de bases de datos y criterios para seleccionar fuentes ligadas a artículos de revistas científicas, tesis doctorales y de magister, publicaciones reconocidas, entre otros:

3.1.1. Estudio del Estado del Arte propio del estatus y desarrollo de las PYMEs.

3.1.2. Estudio del Estado del Arte correspondiente a las Metodologías de Diseño de Modelos Conceptuales y su correlación con el Método Sistémico.

3.1.3. Estudio del Estado del Arte en cuanto a la Gestión Integral de Riesgos, la Inteligencia Decisional, y su aplicación en PYMEs.

3.1.4. Estudio del Estado del Arte en cuanto al Desarrollo de las Componentes de los Sistemas de Gestión QHSE3+: Calidad, Salud y Seguridad, Gestión Ambiental y Eficiencia Energética, al igual que de otros referenciales aplicables, teniendo en cuenta los programas y proyectos de las Comisiones Técnicas de ISO TC, generadoras de las familias de normas internacionales ISO 9000, ISO 45000, ISO 14000, ISO 50000 e ISO 31000.

3.1.5. Estudio del Estado del Arte en cuanto al Desarrollo de la Gestión de Planificación Directiva y Planificación Operacional en los Sistemas de Gestión, y su aplicación en las PYMEs 


\subsection{Configuración del Modelo SIG - QHSE3+}

La configuración del Modelo se realiza de manera global y particular para sus 10 componentes principales, adecuando los desarrollos del Diseño Sistémico que lidera Bernabé Hernandis Ortuño (Hernandis Ortuño \& Briede Westermeyer, 2009) y la Red para el Diseño Sistémico RIS, al caso particular del diseño de un Modelo de Sistemas Integrales de Gestión.

En primera instancia se observa el Sistema como un todo ligado a cada empresa PYME, que tiene unas entradas en términos de recursos, información, condiciones del entorno y requisitos, que se incorporan a la red de procesos de la PYME y generan resultados de negocio, a partir de una Planificación Directiva y Operacional asociadas al Direccionamiento Estratégicoy y a la Gestión Integral de Riesgos. Bajo este enfoque se considera un análisis en cuanto al diseño Estructural, Funcional y Ergonómico (EFE), sobre el Sistema en su globalidad, que posteriormente tiene una réplica en los mismos temas ( $\mathrm{efe}_{\mathrm{i}}$ ) para cada una de las siguientes componentes que lo integran:

a. Núcleo de Dirección: Direccionamiento y Estrategia para el Éxito Sostenible, en integración y simbiósis con la Gestión del Core del Negocio, asociada al Desarrollo de los Productos y Servicios que caracterizan y diferencian la PYME.

b. Corazón del Talento y la Cultura: Gestión de la Cultura, el Desarrollo Organizacional y el Conocimiento. Desarrollo de Competencias. Fortalecimiento del Sentido Social y Humano del Proyecto de Emprendimiento PYME.

c. Coraza de Planificación Operacional, Inteligencia y Riesgos: Este componente correlaciona la Inteligencia y la Gestión Integral de Riesgos con la Planificación Operacional QHSE3+ de los procesos.

A continuación, los componentes $\boldsymbol{d}$ al $\boldsymbol{h}$ conforman los Brazos del Modelo y corresponden a la aplicación de la Planificación Operacional en términos de Buenas Practicas, que le competen a cada uno de los componentes QHSE3, teniendo en cuenta la Estructura de Alto Nivel (HLE por sus iniciales en inglés), definida por ISO para todas las normas sobre Sistemas de Gestión a partir de los procesos de revisión iniciados en el año 2013, que generaron las versiones actualizadas de ISO 9001:2015, ISO 14001:2015 e ISO 45001, en curso de desarrollo para el año 2016.

d. Componente Q - 9k(ISO 9001): Componente correspondiente al Control Operacional aplicado a las Buenas Prácticas asociadas a la Gestión de Calidad de productos, servicios y procesos, focalizadas hacia la prevención de las fallas y no conformidades de sus especificaciones.

e. Componente HS - 45k(ISO 45001): Componente correspondiente al Control Operacional aplicado a las Buenas Prácticas asociadas a la Gestión de Salud y Seguridad, focalizadas hacia la prevención de accidentes de trabajo y enfermedades profesionales.

f. Componente E - 14k(ISO 14001): Componente correspondiente al Control Operacional aplicado a las Buenas Prácticas asociadas a la Gestión Ambiental, que se focaliza en la prevención de la contaminación.

g. Componente E2 - 50K(ISO 50001): Componente correspondiente a la Gestión para la Eficiencia Energética, focalizado en la prevención de los riesgos de uso no racional e ineficiente de los recursos energéticos.

h. Componente Plus (+): Tiene en cuenta el manejo de otro tipo de riesgos específicos que dependiendo del tipo de PYME deben incluirse, como por ejemplo: Riesgos de Inocuidad, de Seguridad de la Información, de Contrabando, Narcotráfico o Comercio ilegal, entre otros.

Los dos últimos componentes del modelo tienen que ver con los elementos que cierran el Ciclo PHVA (Planear, Hacer, Verificar / Realimentar, y Actuar en consecuencia / Mantener, Corregir, Prevenir, Mejorar), propio del contexto integral de calidad de todas las empresas PYME: 
i. Eje de Realimentación: Tiene un alcance que cubre la totalidad de componentes del Sistema y considera los aspectos relacionados con Auditoría, Gestión de Indicadores, Seguimiento, Medición, Evaluación y Análisis, Supervisión, Peticiones, Quejas, Reclamos y Gestión de las Voces de los Grupos de Interés.

j. Eje de Mejora e Innovación: Integra la Gestión de Acciones de Corrección, Tratamiento y Respuesta a No Conformidades e Incidentes, Mejora e Innovación.

\subsection{Configuración de los Instrumentos de Diagnósis y Planificación Directiva y Operacional para el Modelo SIG - QHSE 3+}

En esta fase del flujo de investigación se diseñan los Instrumentos que operacionalizan el modelo para su particularización en términos de Diagnóstico y Planificación, para la configuración efectiva y particularizada del modelo, en función de las características propias de cada PYME.

\subsection{Aplicación y Validación Preliminar de los Instrumentos del Modelo.}

La Validación Preliminar del Modelo tiene en cuenta la aplicación inicial de los Instrumentos de Diagnóstico y Planificación del Modelo y la observación de su pertinencia y beneficios, para la formulación de conclusiones, la declaración de lecciones aprendidas y la proyección de futuras investigaciones.

\section{Revisión Bibliográfica. Aplicación del Protocolo de Estudio del Estado del Arte (E2A).}

Con la aplicación del Protocolo, se desarrollaron las componentes del Estudio del Estado del Arte. De esta manera se obtuvieron las siguientes conclusiones.

\subsection{E2A-1. Estudio del Estado del Arte de las PYMEs:}

No obstante que los Programas de Gobierno y los Planes de Desarrollo de la mayoría de países de occidente tienen en cuenta Desarrollos en Competitividad, Premios y Planes de Incentivos, Programas de Formación, y Planes de Apoyo al Emprendimiento, como se observa en los estudios y las estadísticas del los Ministerios de Desarrollo y Cámaras de Comercio de muchos países de Europa y América, las cifras asociadas al cierre de las PYME se mantienen, y el desarrollo de sus competencias es más limitado en los países en vía de desarrollo, donde además las PYMEs tienen que afrontar una carga fiscal impositiva demasiado alta.

Si bien las condiciones de crisis del petróleo, al igual que la problemática climática y sus implicaciones en las restricciones económicas del mundo en la última década han hecho disminuir el presupuesto asignado, de todas maneras existen varios proyectos de estado, tesis de grado y programas de desarrollo, que desde el sector académico y las organizaciones gremiales aún trazan líneas maestras, para contribuir en la sostenibilidad, el crecimiento y el éxito sostenible de las PYMEs.

Este es el caso de los estudios de (Marcelino-Sádaba, et al., 2014), y (Del Caño \& De La Cruz, 2002), por sus trabajos en materia de Riesgos para estas organizaciones, como de (Gomez y Rialp, 2008), (Madrid y García, 2008), (Rabentino 2005) y (Zornoza, 2000), quienes en sus investigaciones muestran los desafíos, el imperativo y las tendencias del presente y futuro de las PYMEs, como se ha resumido en el anterior párrafo. 


\subsection{E2A-2. Estudio del Estado del Arte del Diseño de Modelos Conceptuales y su correlación con el Método Sistémico.}

El Diseño de Modelos Conceptuales y la aplicación de la Sistémica y la Ingeniería Concurrente para su enfoque en todo tipo de productos, servicios, organismos y sistemas se ha venido desarrollando a partir de los desarrollos de la Teoría General de Sistemas (Bertalanffy, 1975) y posteriormente con escuelas particulares de diseño que han integrado el diseño gráfico con el diseño en sus componentes funcional, ergonómico y formal, y a partir del enfoque sistémico, el diseño para estos tres componentes, modelando sobre objetivos de explotación, gestión, evolución y mutación. Este es el caso particular de la Red Latina de Diseño, que liderada por Bernabé Hernándis Ortuño (Cardozo J., Hernandis B., et al, 2015) y desde la revista rdis "revista de la red internacional de investigación en diseño", ha venido integrando los trabajos y esfuerzos de las escuelas de diseño y los investigadores de Argentina, Brasil, Chile, Colombia, España, Italia, México y Venezuela, entre otros.

\subsection{E2A-3. Estudio del Estado del Arte de la Gestión Integral de Riesgos.}

En su componente de Inteligencia y Gestión Integral de Riesgos, el modelo se ha fundamentado en los desarrollos de las últimas dos décadas que ha liderado la escuela australiana y neozelandesa, a través de los Comités de Normalización de AS/NZ e ISO, quienes dinamizaron el desarrollo de la norma ISO 31000:2009, “Administración Integral de Riesgos”, y las demás normas que integran su familia, en temas ligados a la estimación del riesgo, los indicadores, la auditoría y los indicadores para la gestión del riesgo. Esta dinámica se complementa con el Enfoque de Inteligencia del Departamento de Seguridad Nacional Norteamericano y de la Policía de las Américas (USA - DHL, 2010), y con los desarrollos en materia de Control Interno desde el modelo del "Committee of Sponsoring Organizations of the Treadway Commission" (COSO).

\subsection{E2A-4. Estudio del Estado del Arte de la Gestión QHSE3+}

Esta componente del E2A se nutre de los desarrollos de las familias de Normas ISO 9000, desde el Comité ISO TC 176; ISO 45000, con el Comité PC 283; ISO 45000 con el Comité TC 207 e ISO 50000 bajo el Comité TC 242. Desde cada una de estas comisiones, y bajo un Plan de Desarrollo particular de cada una se van jalonando los desarrollos en estos temas.

\subsection{E2A-5. Estudio del Estado del Arte de la Gestión de Planificación Directiva y la Planificación Operacional en las PYMEs}

Los desarrollos de las Escuelas de Negocios y Administración en Materia de Planificación Directiva y Estratégica, al igual que el enfoque de procesos y su aplicación en transversal en cuanto a definición de procesos, especificaciones y controles constituyen los elementos de Planificación que nutren en el modelo, bajo el desafío del éxito sostenible para las PYME.

\section{Desarrollo. Argumentación y Resultados Obtenidos}

\subsection{El Modelo SIG - QHSE3+:}

En la figura 3 se observa el modelo configurado, en el que se articulan los componentes descritos en la sección 3, que tiene una Estrella como Núcleo de Dirección, seguido del Corazón que representa el Talento y la Cultura, con una Coraza de tres capas que reúnen la Inteligencia, la Gestión de Riesgos y la Planificación Operacional. A continuación, el modelo plantea 5 Brazos, para las componentes QHSE3+, y su estructura culmina con dos Ejes de Dinámica, que corresponden al Eje de Realimentación y el Eje de Mejora e Innovación. 
Desde un punto de vista general se destaca que para el Modelo se ha considerado el estudio de las componentes de Diseño Funcional, Ergonómico y de Forma (FEF), al tiempo que, de manera particular para cada uno de los diez elementos señalados en el párrafo anterior (fef), se hace la réplica de este análisis, destacando el enfoque sistémico y la determinación de entradas, procesos relacionados, salidas y métricas ligadas a sus objetivos.

\subsection{Los Instrumentos de Diagnósis y Planificación.}

Una vez realizado el diseño gráfico y Funcional/Estructural, Ergonómico y Formal del Sistema Integral de Gestión SIG-QHSE3+, se ha procedido a configurar varios instrumentos básicos que facilitan su configuración, comprensión y puesta a punto para la operación. Estos instrumentos se dividen básicamente en dos bloques:

\subsubsection{Instrumentos para la comprensión y el Diagnóstico del SIG-QHSE3+,}

Estos instrumentos están reunidos como un libro Excel que plantea una hoja Excel para cada uno de los 7 Principios Universales de Gestión Integral: a) Enfoque en las Partes Interesadas, b) Liderazgo Gerencial, c) Humanización y Desarrollo del Talento, d) Enfoque de Procesos, e) Enfoque Basado en Hechos para la toma de decisiones, f) Relaciones mutuamente beneficiosas con los grupos de interés, y g) Mejora Continua.

En cada una de las hojas y para cada principio se realiza una calificación de 10 preguntas que cubren el ciclo PHVA aplicado a los riesgos Q(ISO 9001/Calidad), HS(ISO 45000/Salud y Seguridad), E(ISO 14001/Gestión Ambiental, y E2(ISO 50001/Eficiencia Energética).

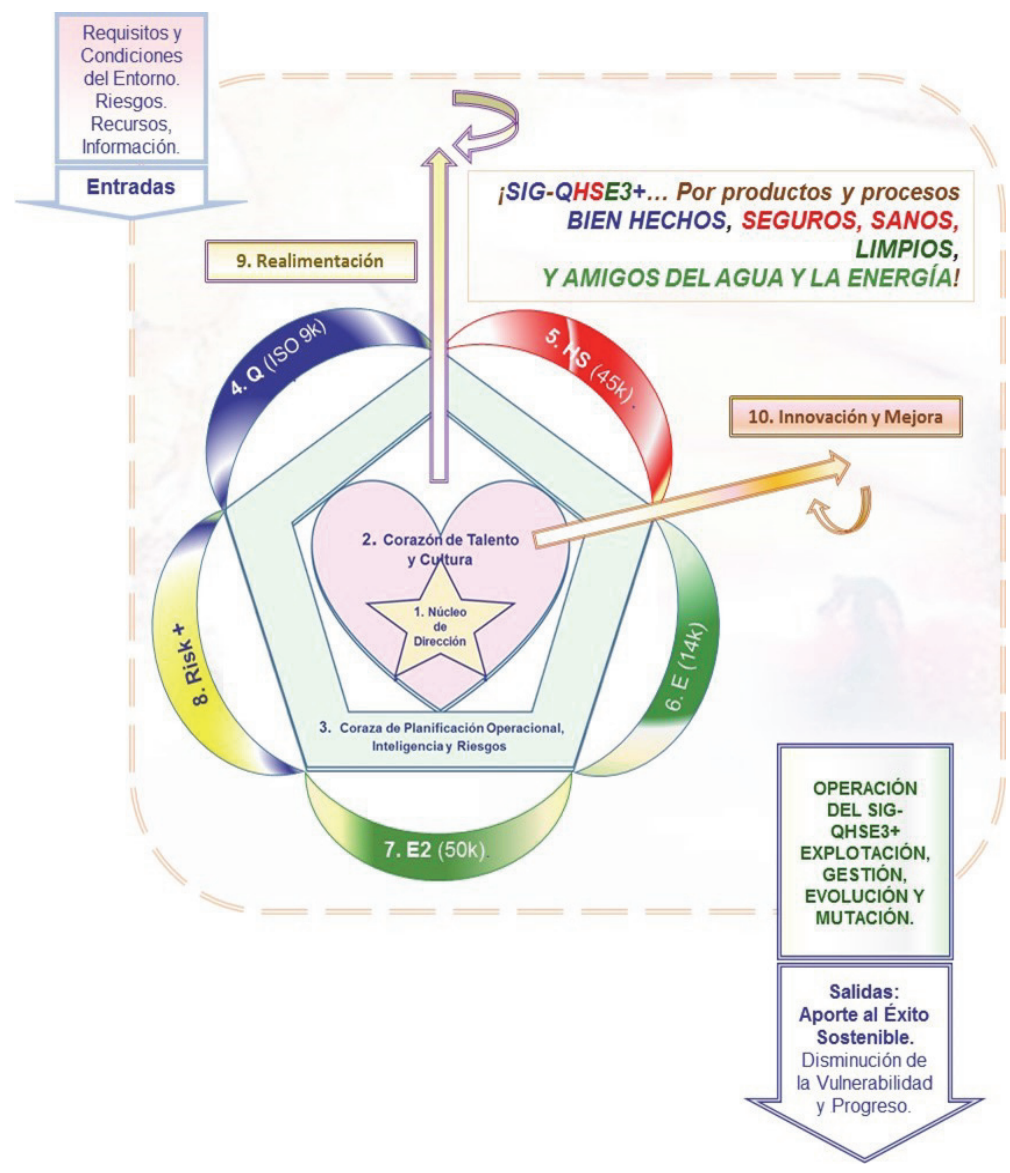

Fig. 3 Boceto de Representación del Modelo SIG-QHSE3+. Fuente: Poveda Orjuela (2016). 
Se deja abierta la posibilidad de formular una o varias columnas adicionales para referenciales ligados a especificaciones adicionales que apliquen según la naturaleza de los productos, los procesos y las empresas relacionadas.

La aplicación de estos instrumentos permite obtener una línea base y establecer prioridades particulares para la configuración detallada del modelo.

\subsubsection{Instrumentos para la Planificación Directiva.}

Estos instrumentos permiten soportar la Planificación Directiva del Sistema y del Negocio, a partir de la definición del contexto vs los productos o servicios clave de las PYME, la definición del Modelo de Negocio, los propósitos y objetivos estratégicos, los riesgos críticos del negocio bajo un análisis de motricidad, dependencia que aplica el enfoque de la escuela Prospectiva (Godet, 2000), en conjugación con el análisis clásico de importancia y gobernabilidad, para formular posteriormente los proyectos y medidas de administración de estos riesgos, y la Política Integral de Gestión SIG-QHSE3+,

\subsubsection{Instrumentos para la Planificación Operacional y la Gestión Integral de Riesgos QHSE3+}

El enfoque de procesos se materializa en el modelo a partir de la Planificación de su secuencia, la identificación de riesgos en cada una de sus componentes QHSE3+, y la definición de medidas integrales de prevención, control, medición y seguimiento por cada proceso. En este punto se centra la Gestión del Modelo en cuanto al Pensamiento Integral basado en riesgos.

El enfoque de la Planificación Operacional puede considerar la formulación de Planes particulares QHSE3+ en la configuración de Proyectos Clave, o bien en la Planificación, Diseño y Desarrollo de Productos y Servicios bajo el enfoque $\mathrm{i}+\mathrm{d}+\mathrm{D}+\mathrm{I}$, según sea el caso.

\section{Conclusiones}

Con la aplicación de las líneas metodológicas expuestas de manera general en las anteriores secciones, se logró poner al servicio de los emprendedores de las PYMEs un Modelo Integral para los Sistemas Integrales de Gestion que mediante Instrumentos montados sobre hojas electrónicas básicas, permite llenar el vacío actual en este sentido y complementar los pocos instrumentos específicamente diseñados para las PYMEs que se encuentran disponibles para el público en general, entre los que se destacan principalmente los trabajos de (Marcelino-Sádaba, et al., 2014), y (Del Caño \& De La Cruz, 2002), que aplican para el caso particular de Gestión de Riesgos en Proyectos para el sector de la construcción, pero no tienen en cuenta la visión integral SIG-QHSE3+, ni la problemática de los Sistemas de Gestión de las PYMEs.

Se plantean a continuación, a título de conclusión general, los puntos de innovación, los aportes, y los logros específicos generados:

a) La apropiación, particularización y validación de un modelo de trabajo sencillo que facilita aplicar en cada PYME los ciclos y etapas para la configuración y operación de los SIG-QHSE3+, bajo un enfoque de Prevención fundamentado en la aplicación de las Buenas Prácticas y la Inteligencia de la Información para la Toma de Decisiones acertadas.

b) El desarrollo y validación de Instrumentos de Comprensión y Diagnóstico del ámbito del SIG, que soportan también la particularización de su configuración, al tiempo que su Planificación Directiva y Operacional focalizada en los negocios PYME. 
Aplicación del método sistémico al diseño de un modelo conceptual para sistemas integrales de gestión QHSE3+ en PYMES.

c) La aplicación sistemática e integral (QHSE3+,) del Pensamiento Basado en Riesgos desde el análisis del problema y las necesidades asociadas, en la estructuración de los Bancos de Ideas, de Conceptos y en la Administración del Portafolio de Proyectos, al igual que en el ciclo transversal de Direccionamiento Estratégico, Enfoque de Procesos y Operación de los negocios PYME.

d) La incorporación del análisis de Motricidad y Dependencia, como herramienta para soportar las decisiones en cuanto a la priorización de los frentes preventivos de acción ante riesgos y problemas, y como instrumento de análisis de las prioridades que tienen en campo los diferentes elementos directivos y operacionales de los negocios PYME.

e) La focalización en mapas de riesgos y la incorporación del Pensamiento Basado en Riesgos a la cotidianidad operacional y estratégica de los directivos, responsables de la gestión del emprendimiento y los procesos de las PYME, cuando al lograr disminuciones porcentuales de vulnerabilidad del riesgo general entre el 15\% y el 37\%, que pueden llegar a representar cifras mayores de reducción de costos; entienden y apropian la ecuación (1):

\section{CALIDAD INTEGRAL = APLICACIÓN DE BUENAS PRÁCTICAS $=$ GESTIÓN DE RIESGOS $=\%$ DISM. DE LA VULNERABILIDAD =\%DISMIN. COSTOS}

$$
\% \text { DismVul }=\left[\sum_{i=1}^{n}\left(P o_{i}\right) \cdot\left(G o_{i}\right)-\sum_{i=1}^{n}\left(P f_{i}\right) \cdot\left(G f_{i}\right)\right] /\left[\sum_{i=1}^{n}\left(P o_{i}\right) \cdot\left(G o_{i}\right)\right]
$$

Donde DismVul corresponde al porcentaje de Disminución de la Vulnerabilidad, luego de aplicar las Buenas Prácticas, Poi y Goi representan la valoración inicial de la Posibilidad y la Gravedad de cada riesgo, en tanto que Pfi y Gfi corresponden a la Posibilidad y Gravedad finales, después de haber puesto en aplicación las medidas de prevención asociadas a las Buenas Prácticas.

f) La apertura y generación de campos para nuevas investigaciones y proyectos PYME con herramientas sectoriales para los diferentes tipos de empresas PYME y proyectos de desarrollo de nuevos productos y negocios, emprendimiento, inversiones y desarrollo urbano, social, tecnológico e industrial.

\section{Referencias Bibliográficas}

ACEDO F., (2003). "Los factores subjetivos e institucionales en el proceso de internacionalización de la empresa", Tesis Doctoral, Universidad de Sevilla.

ANDERSSON, S. (2000). "The Internationalization of the Firm from an Entrepreneurial Perspective", en International Studies of Management and Organization, Vol. 30, n. ${ }^{\circ}$ 1, pp 63-92.

ANDER-EGG, E., (2013). “Rethinking Participatory Action Research”. 4th edition 157pp ed. Buenos Aires: Lumen Humanitas. ISBN: 9789870003779.

BENAVENT, G., (2012). “Metodología de Valoración de Alternativas de Diseño basada en Modelos Sitémicos”, en Revista RDIS. Volumen 1, Número 2, P8-15.

BERTALANFFY, L. (1975): "Perspectivas en la teoría general de sistemas", en Tendencias en la teoría general de sistemas. Madrid: Alianza Universidad, 1978.

BONILLA J. y ALEGRE J., (2012). “Empresas Gacelas: definición y caracterización”, en Academia. Revista Latinoamericana de Administración Número 50, 2012, pp. 31-43. 
BRIEDE J., (2010). "La Metodología Sistémica y el Rol de las Representaciones en el Diseño Conceptual de Productos Industriales”, en Umbral Científico, Número 17, 2010, pp. 73-82.

CARDOZO J., HERNANDIS B. y RAMÍREZ N., (2015). "Aproximación a una categorización de los sistemas de productos: el uso y la experiencia del consumidor como configuradores”, en Innovar, Volumen 25, Número 58, pp. 125-142. doi: 10.15446/innovar. Bogotá.

CHECKLAND P. (1981), "System thinking, systems practice”, Chichester: John Wiley \& Sons

DEL CAÑO, A. \& DE LA CRUZ, M. D. P., (2002). "Integrated Methodology for Project Risk Management” en Journal of Construction Engineering and Management, December, 128(6), pp. 473-485.

FEDERICO, J.; KANTIS, H. y RABETINO R., 2009: “Factores determinantes del crecimiento en empresas jóvenes. Evidencias de una comparación internacional", en Capelleras, J. y Kantis, H. (Coord.) : "Nuevas empresas en América Latina: factores que favorecen su rápido crecimiento", Universidad Autónoma de Barcelona, Universidad Nacional de General Sarmiento y AECID (Ministerio de Asuntos Exteriores y Cooperación de España). http://www.rabetino.com/publicaciones.html

GOMEZ J. y RIALP J. 2008. "Influencia de la orientación al mercado en la función empresarial: su impacto en la capacidad de innovación” en Revista Internacional de la Pequeña y Mediana Empresa Vol. I, Número 1, pp. 125-126

GODET, M., 2000. "The Art of Scenarios and Strategic Planning: Tools and Pitfalls” en Technological Forecasting and Social Change, 65(1), pp. 3-22.

HERNANDIS ORTUÑO, B, BRIEDE-WESTERMEYER, J. C., CABELLO MORA, M. \&., (2014). “Concurrent sketching model for the industrial product conceptual design" en DYNA, 81(187), pp. 199-208.

HERNANDIS ORTUÑO B. e IRIBARREN E. , (2011). “Modelización de Sistemas”. Grupo de investigación y Gestión del Diseño, Universitat Politècnica de València.

HERNANDIS ORTUÑO, B., (2004). "Diseño de nuevos productos, una perspectiva sistémica”. Universidad Politecnica de Valencia Universitat Politècnica de València.

HERNANDIS ORTUÑO, B., (2003). "Desarrollo de una Metodología Sistémica para el Diseño de Productos Industriales”. Tesis Doctoral no publicada. Valencia: Universitat Politècnica de València.

HERRERA J., (2005). “Aplicación del enfoque sistémico en el diseño de los sistemas de transporte ferroviario de carga”, en Ingeniería. Investigación y Tecnología, vVol. 6, Número 4, octubre-diciembre, 2005, pp. 299-309 Universidad Nacional Autónoma de México Distrito Federal, México.

INTERNATIONAL ORGANIZATION FOR STANDARDIZATION, (2009). "Risk Management. Principles and Guidelines". ISO 31000:2009. Geneve: ISO.

INTERNATIONAL ORGANIZATION FOR STANDARDIZATION, (2012). "Guidance on Project Management". ISO 21500:2012. Geneve: ISO.

INTERNATIONAL ORGANIZATION FOR STANDARDIZATION, 2015. "Quality management systems. Requirements with guidance for use". ISO 9001: 2015-A. Geneve: ISO.

INTERNATIONAL ORGANIZATION FOR STANDARDIZATION, (2015). "Environmental management systems. Requirements" ISO 14001: 2015-B. Geneve: ISO.

INTERNATIONAL ORGANIZATION FOR STANDARDIZATION, (2009). "Risk Managemnet". ISO 31000:2009, SA/SNZ HB 436:2013. Sidney Diciembre 16 2013: SAI Global limited ISBN 0781743426333.

MADRID A. y GARCÍA D, (2008). "Las ayudas financieras a la innovación a la PYME. Sesgo de motivación y de selección administrativa, en Internacional Review of Small and Medium Enterprise”, Volumen 1, Número 1, pp 1736

MARCELINO-SÁDABA, S., PÉREZ-ESCURDIA, A., ECHEVERRÍA LAZCANO, A. M. \& VILLANUEVA, P., (2014). "Project risk management methodology for small firms" en International Journal of Project Management, Issue 32, pp. 327-340.

MARTÍNEZ E., (2007). "La cultura organizacional y la implantación de las tecnologías de la información" AUTOR. Tesis Doctoral Universidad Politécnica de Cartagena, Departamento de Economía de la Empresa.

MOOTEE, I. (2014). "Design Thinking for Strategic Innovation. What They Can't Teach You, at Business or Design School”. Barcelona, España: Ediciones Urano S.A. 
POVEDA ORJUELA, P. P., CAÑÓN ZABALA, G., (2015-A.) "Guía para la Gestión Integral de Riesgos. Comprender, decidir y actuar con inteligencia para el éxito sostenible”. Bogotá D. E.: ICONTEC. ISBN 978-9588585-51-2.

POVEDA ORJUELA, P. P., CAÑÓN ZABALA, G. (2015). "Herramientas para implementar un Sistema de Gestión de Calidad según ISO 9001”. 4a ed. en pre-prensa. Bogotá: ICONTEC. ISBN 958.33.9032-1.

RABENTINO R., (2005). "Factores determinantes del crecimiento de las PYMEs latinoamericanas” Tesis Doctoral, Barcelona, Universidad Autónoma de Barcelona.

SAI, STANDARDS AUSTRALIA INTERNATIONAL LTD AND SNZ, STANDARDS NEW ZEALAND. (2013). "Handbook Risk Management Guidelines - Companion to AS / NZS

USA - DHL, (2010). “DHS Risk Lexicon. NPPD Risk Management \& Analysis”. 2010 Edition ed. Washington: Homeland Security Office.

ZORNOZA C., (2000). "Reflexiones sobre la Investigación Cientifica en las PYME", en Investigaciones Europeas de Dirección y Economía de la Empresa Vol. 6, Número 2, pp. 13-30. 